\title{
Reflections on 24 years of the NSW Public Health Bulletin
}

\author{
Sarah Thackway ${ }^{\mathrm{A}}$, Kristy M. Goldsworthy ${ }^{\mathrm{A}, \mathrm{B}, \mathrm{D}}$, \\ Beth Stickney ${ }^{\mathrm{A}, \mathrm{C}}$ and Andrew J. Milat ${ }^{\mathrm{A}, \mathrm{C}}$ \\ ${ }^{\mathrm{A}}$ Centre for Epidemiology and Evidence, NSW Ministry of Health \\ ${ }^{\mathrm{B}}$ Editorial Manager, NSW Public Health Bulletin \\ ${ }^{\mathrm{C}}$ Editor, NSW Public Health Bulletin \\ ${ }^{\mathrm{D}}$ Corresponding author.Email: kgold@doh.health.nsw.gov.au
}

\section{The birth of the Bulletin}

The year 1990 was a landmark for public health in New South Wales (NSW). The Public Health Division at the then NSW Department of Health was in its infancy, having been formed at the end of 1989 , and funding for a program to enhance public health in NSW led to the establishment of a network of Public Health Units and the NSW Public Health Officer Training Program. ${ }^{1}$ The NSW Public Health Bulletin was established in May 1990 to disseminate accurate and timely information among this newly formed public health network and to provide regular feedback to practitioners on notifiable conditions. It was hoped that the Bulletin would assist in "the development of a vibrant public health network in NSW", 2 and "provide a useful mechanism for exchange of information and ideas on investigations, programs, and evaluations that (may) affect the health of the citizens of NSW". ${ }^{1}$

From its inception, the aim of the Bulletin was to provide its readers with population health information and data to inform effective public health action. Early editions were of variable length (although generally short) and consisted of practical information for the public health network: short reports, news and comment, letters to the Editor, summaries of infectious disease notifications, and public health abstracts. Copies were distributed in collaboration with the new Public Health Units to the then Area Health Services, hospitals, major laboratories, universities, medical practitioners, and other state health departments.

\section{Enhancement of content, rigour, role and recognition}

By 1995, the landscape was changing: issues of the Bulletin were becoming longer and the papers more academically rigorous. Infectious disease content still featured strongly but a broader range of topics was being covered. Special editions focusing on a specific area of public health significance commenced, with guest editorials authored by experts in the field. Peer review was introduced to maintain standards and ensure rigour and relevance. At the beginning of 1996, the Bulletin began to be published in an online format as well as in print.

The transformation of the Bulletin in its first decade culminated in the successful application in early 2002 to the Literature Selection Technical Review Committee (LSTRC) of the National Library of Medicine, National Institutes of Health (Maryland, USA) for indexing in Index Medicus and Medline. As the major international source of citation and the gateway to public health and biomedical journals, the decision by the LSTRC to accept the Bulletin for indexing was to be celebrated. At the time, around 120 journals were reviewed each year by the LSTRC and only $15-20 \%$ of those were successful in their applications to be accepted for indexing. Criteria for acceptance related to: scope and coverage (relevance to the biomedical field); quality of content (scientific merit of the papers); quality of the editorial work (including processes such as peer review); production quality (layout, design and graphics); audience (intended for health professionals); and types of content (with statistical compilations and critical reviews preferred). ${ }^{3}$ A survey of a sample of the Bulletin's readership in 2005 indicated that the inclusion of the Bulletin in Medline was highly valued:

[It] is seen as a measure of the journal's quality and thereby adds status to the Bulletin; increases the accessibility and international exposure for articles published; and helps showcase public health issues and endeavours in NSW. ${ }^{4}$

The 2005 readership review also found substantial support and respect for the Bulletin, along with a strong sense of 'ownership' amongst its stakeholders. ${ }^{4}$ A valued aspect was the workforce development role of the Bulletin: it was not just another avenue for established authors to publish, but an opportunity for those new to writing for publication to develop their skills in a supportive environment.

\section{Improved reach, processes and promotion}

In 2007 the Bulletin entered into a publishing partnership with CSIRO Publishing. This was the next phase in the Bulletin's evolution, taking it to a larger, more professional publication with a broader reach. A new design was unveiled, and an enhanced website hosted by CSIRO Publishing was launched. The new website allowed for PDF and HTML versions to be uploaded in advance of the hard copy distribution and for the introduction of a 
subscription-based 'Early Alert' service to facilitate immediate access to each new issue as it was published online. New resources were developed for reviewers, including guidelines designed to foster the development of critical peer review skills and a standard reviewer report form to guide reviewers through the process. Guidelines for authors were also updated to reflect best publishing practice. The partnership with CSIRO Publishing also led to increased exposure and promotion at relevant health conferences. Free access, a cornerstone of the publication, was preserved: work published in the Bulletin remained freely available, without any subscription barriers. This will continue to be the case as the Sax Institute takes over from CSIRO Publishing as the publisher of the journal. ${ }^{5}$

\section{Achievements and contributors}

Of the past Editors of the Bulletin, three were largely responsible for its growth and evolution: Professor George Rubin (May 1990-May 1994), Professor Michael Frommer (June 1994-March 1998), and the longest running Editor, Associate Professor Lynne Madden (April 1998October 2012, with two sabbaticals in this period). While ultimately rewarding, the role of Editor is a challenging one; the past Editors are gratefully acknowledged for their commitment and contribution to the Bulletin. There have been many editorial support staff during the Bulletin's lifetime, the longest serving of these being Dr Michael Giffin (November 1998-January 2005), who played an integral role in shaping the Bulletin, establishing many of its editorial processes, and securing indexing in Index Medicus and Medline.

At the completion of Volume 24, close to 1500 items have been published and an average of 220000 papers are downloaded per year. These downloads are from all volumes, indicating that the Bulletin, as a body of work, is contributing to a culture of evidence-based practice. The Bulletin's Scopus SCImago Journal Rank (SJR), which measures citations to a publication and weights them according to the SJR score of the citing journal, has been rising since 2008, showing that the Bulletin is well positioned in relation to comparable journals. While the majority of the readership remains local, download and access statistics show that the Bulletin is read widely: within Australia, readership from Victoria and Queensland is strong, and internationally, numbers from the USA, Canada and the United Kingdom are consistent.

On behalf of the editorial team we would like to sincerely thank the many contributors to the Bulletin over the past 24 years. The strength of the journal has always been the large investment of time and effort by authors, reviewers, and editors, all of whom have generously contributed their expertise.

\section{The future: Public Health Research \& Practice}

As we enter the next exciting phase in the journal's history, ${ }^{5}$ we hope that the 24 volumes published under the title 'NSW Public Health Bulletin' leave a lasting legacy and will continue to be referred to, both as an important historical record of public health in NSW and as sources of evidence for future practice.

\section{References}

1. Rubin G. Improved public health. $N S W$ Public Health Bull 1990; $1: 2$.

2. Morey S. New Public Health Bulletin for NSW. N S W Public Health Bull 1990; 1: 1 .

3. Madden D, Giffin M. Index Medicus and Medline recognise NSW Public Health Bulletin. N S W Public Health Bull 2002; 13: 1-2. doi:10.1071/NB02001

4. Madden DL. Tailoring the Bulletin to meet readership needs. N S W Public Health Bull 2006; 17(11-12): 161-2.

5. Thackway S, Redman S. Transition of the NSW Public Health Bulletin to Public Health Research and Practice. N S W Public Health Bull 2014; 24(4): 149-50. 